\title{
Formation of steep, low Schottky-barrier contacts by dopant segregation during nickel silicidation
}

S. F. Feste, J. Knoch, D. Buca, Q. T. Zhao, U. Breuer, and S. Mantl

Citation: Journal of Applied Physics 107, 044510 (2010);

View online: https://doi.org/10.1063/1.3284089

View Table of Contents: http://aip.scitation.org/toc/jap/107/4

Published by the American Institute of Physics

\section{Articles you may be interested in}

Tuning the Schottky barrier height of nickel silicide on $p$-silicon by aluminum segregation

Applied Physics Letters 92, 222114 (2008); 10.1063/1.2940596

Effective Schottky barrier lowering in silicon-on-insulator Schottky-barrier metal-oxide-semiconductor field-effect transistors using dopant segregation

Applied Physics Letters 87, 263505 (2005); 10.1063/1.2150581

Tuning of NiSi / Si Schottky barrier heights by sulfur segregation during Ni silicidation Applied Physics Letters 86, 062108 (2005); 10.1063/1.1863442

Evidence for strong Fermi-level pinning due to metal-induced gap states at metal/germanium interface Applied Physics Letters 91, 123123 (2007); 10.1063/1.2789701

Fermi level depinning and contact resistivity reduction using a reduced titania interlayer in $\mathrm{n}$-silicon metalinsulator-semiconductor ohmic contacts

Applied Physics Letters 104, 112101 (2014); 10.1063/1.4868302

The physics and chemistry of the Schottky barrier height

Applied Physics Reviews 1, 011304 (2014); 10.1063/1.4858400

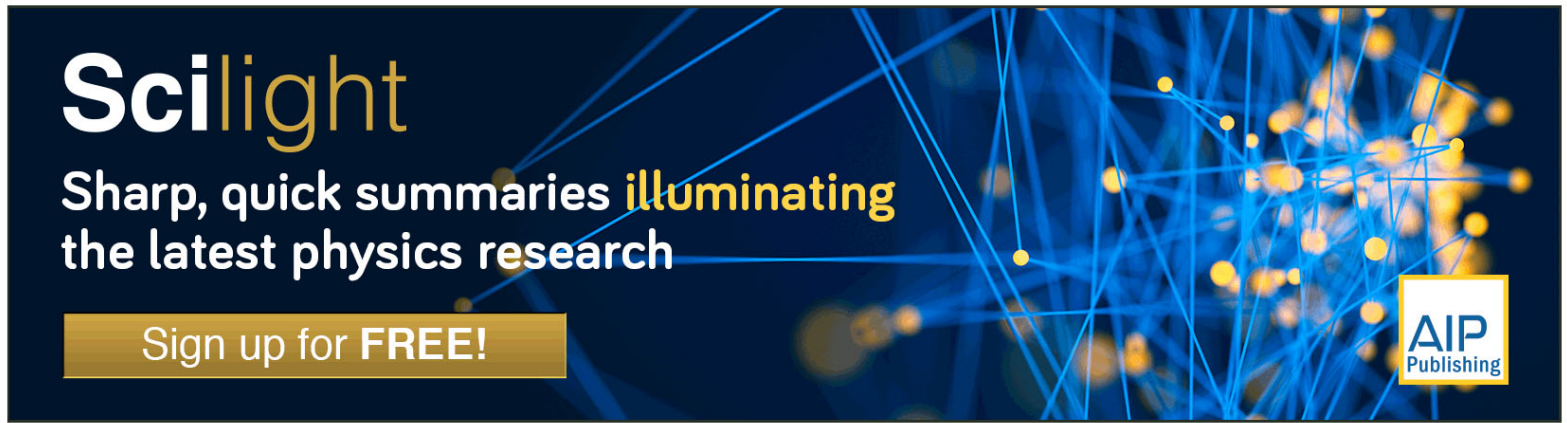




\title{
Formation of steep, low Schottky-barrier contacts by dopant segregation during nickel silicidation
}

\author{
S. F. Feste, ${ }^{1, a)}$ J. Knoch, ${ }^{2}$ D. Buca, ${ }^{1}$ Q. T. Zhao, ${ }^{1}$ U. Breuer, ${ }^{3}$ and S. Mantl ${ }^{1}$ \\ ${ }^{1}$ Institute of Bio- and Nanosystems (IBN1) and JARA-FIT Fundamentals of Future Information Technology, \\ Forschungszentrum Jülich, 52425 Jülich, Germany \\ ${ }^{2}$ Micro- and Nanoelectronics Devices Group, TU Dortmund University, 44227 Dortmund, Germany \\ ${ }^{3}$ Central Division of Analytical Chemistry (ZHC), Forschungszentrum Jülich, 52454 Jülich, Germany
}

(Received 27 July 2009; accepted 4 December 2009; published online 24 February 2010)

\begin{abstract}
We present a systematic analysis of arsenic dopant segregation during nickel silicide formation. The slopes and concentrations of the arsenic dopant profiles at the $\mathrm{NiSi} / \mathrm{Si}$ interface have been studied as a function of implantation energy, implantation dose, and NiSi thickness. Silicidation induced dopant segregation conserves the dopant slope at the silicide/silicon interface up to NiSi thicknesses of three times the as-implanted peak depth before degrading. Best slopes and highest dopant concentrations are obtained for low implantation energies and thin NiSi layers. We also demonstrate that the steepness of the dopant profile at the $\mathrm{NiSi} / \mathrm{Si}$ interface can be significantly improved through a two-step annealing process for NiSi formation. For As, $1 \mathrm{keV}, 1 \times 10^{15} \mathrm{~cm}^{-2}$, and a $17 \mathrm{~nm}$ $\mathrm{NiSi}$ layer, a NiSi/Si junction with a dopant slope of $3.2 \mathrm{~nm} /$ decade has been obtained. An effective Schottky barrier of $\Phi_{\mathrm{SB}}=0.12 \mathrm{eV}$ was determined by low temperature measurements of Schottky diodes with $20 \mathrm{~nm}$ NiSi formed by an optimized annealing process. (C) 2010 American Institute of Physics. [doi:10.1063/1.3284089]
\end{abstract}

\section{INTRODUCTION}

Recently, there has been a renewed interest in Schottky barrier (SB)-MOSFET as an alternative to conventional metal-oxide-semiconductor field-effect transistors (MOSFETs) which require highly conductive contacts with abrupt doping profiles when scaled to ultimately small channel lengths. ${ }^{1,2}$ Metallic source/drain contacts in SBMOSFETs ensure lower parasitic resistances compared to conventional doped contacts. NiSi is one of the most attractive choices for the integration into ultrathin-body devices due to its low $\mathrm{Si}$ consumption and low resistivity. NiSi contacts with sheet resistances of as low as $10^{-6}-10^{-7} \Omega / \square$ can be achieved on both $\mathrm{p}$ - and n-type $\mathrm{Si}^{3}{ }^{3}$ However, due to the SB $\left(\Phi_{\mathrm{B}}\right)$ at the metallic source/drain contacts, the performance of SB-MOSFETs still falls behind that of conventional FETs. ${ }^{4}$ Therefore, much work was devoted to lower the SB height $(\mathrm{SBH})$ by using silicides with lower $\Phi_{\mathrm{B}}{ }^{5,6}$ or dopant segregation ${ }^{7-10}$ and to improve the carrier injection into the channel by using thin gate oxides and thin-body silicon-on-insulator (SOI). ${ }^{11,12}$ Recently, short-channel p-type SB-FETs with NiSi and PtSi contacts and boron dopant segregation have been demonstrated that show oncurrents comparable to conventional unstrained SOI FETs. ${ }^{13,14}$

Traditionally, an Ohmic contact between a semiconductor and a metal is formed by highly doping the semiconductor. ${ }^{15}$ However, for a fully depleted SOI MOSFET channel doping causes merely a shift of the threshold voltage and reduced carrier mobility. The carrier injection into the channel remains unaffected, and therefore subthreshold swing and transconductance are not improved. In order to

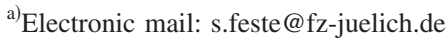

increase the carrier injection without shifting the threshold voltage, a nonuniform doping profile with a high dopant concentration only at the metal/semiconductor interface is needed. ${ }^{16}$ This can be achieved through silicidation induced dopant segregation. ${ }^{17}$ The segregation layer causes a strong band bending at the contact/channel interface, effectively thinning the SB [Fig. 1(a)]. However, for application in advanced complementary metal oxide semiconductor (CMOS) technology, it is essential that devices based on dopant segregation are scalable to ultrashort-channel lengths. As the channel length is decreased, source and drain contacts come closer together and the dopant tails of the segregation layers overlap [Fig. 1(b)]. There is a minimum channel length, $L_{\min }$, at which the overlap of these tails starts to constitute a significant channel doping that gradually leads to a loss of improved carrier injection and degraded mobility. The value of $L_{\min }$ is determined by the extent of the segregation layer, $l_{\text {seg, }}$, and the slope of the dopant tail into $\mathrm{Si}$, schematically shown in Fig. 1(b). Hence, thin segregation layers with very steep dopant profiles into $\mathrm{Si}$ at the contact/channel interface are required.
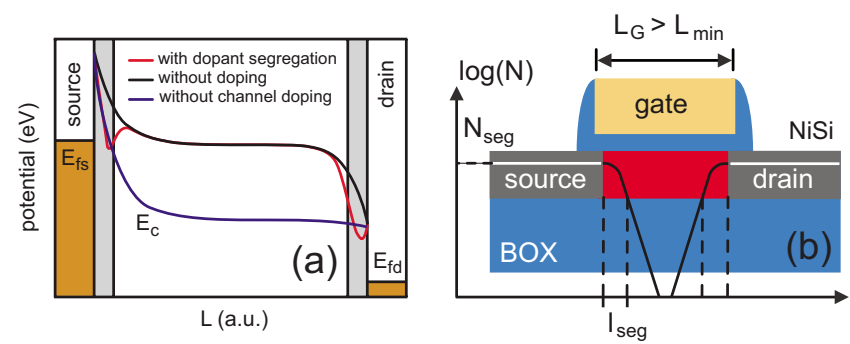

FIG. 1. (Color online) (a) Schematic conduction band profile for SB-FETs without doping, with channel doping, and with dopant segregation. (b) With decreasing channel length $L$, the dopant dopant tails of the segregation layers overlap and constitute a dopant concentration in the channel. 
In this work, we present a systematic investigation of arsenic dopant segregation during nickel silicidation, with emphasis on process compatibility with MOSFET downscaling requirements for nanoscale SB-FETs. To this end, we focus on the dependence of the slope of the dopant profile into $\mathrm{Si}$ and the dopant concentration at the $\mathrm{NiSi} / \mathrm{Si}$ interface on implantation energy, NiSi thickness, and the annealing process employed for the formation of the silicide layer. Moreover, we demonstrate by low temperature measurements on Schottky diodes that an optimized annealing process for NiSi formation after implantation is effective in lowering the $\mathrm{NiSi} / \mathrm{Si} \mathrm{SBH}$ down to a value of $\Phi_{\mathrm{B}}=0.12 \mathrm{eV}$.

\section{SAMPLE PREPARATION}

Several sets of junctions have been fabricated on device grade (001) Si and SOI substrates: samples were implanted with As to doses between $5 \times 10^{13}$ and $1 \times 10^{15} \mathrm{~cm}^{-2}$ at energies from 1 to $10 \mathrm{keV}$. After cleaning and removing the native oxide with (1\%) HF solution, Ni layers of different thicknesses were deposited by electron beam (e-beam) evaporation. Subsequently, the samples were annealed at $450{ }^{\circ} \mathrm{C}$ for $30 \mathrm{~s}$ in forming gas to form nickel monosilicide (NiSi). Unreacted $\mathrm{Ni}$ was removed with $\mathrm{H}_{2} \mathrm{SO}_{4}: \mathrm{H}_{2} \mathrm{O}_{2}$ (4:1) solution. Furthermore, junctions with two modified processes were fabricated: in the first process, after As implantation, $\mathrm{Ni}_{2} \mathrm{Si}$ was formed at $280^{\circ} \mathrm{C}$ for $90 \mathrm{~s}$, followed by the removal of the unreacted metal and a second annealing at $450{ }^{\circ} \mathrm{C}$ to form NiSi. In the second process, a $\mathrm{Ni}_{2} \mathrm{Si}$ layer was implanted with As and transformed into NiSi afterward.

The dopant profiles of the as-implanted and silicided samples were measured with time-of-flight secondary ion mass spectroscopy (SIMS) using a $1 \mathrm{keV} \mathrm{Cs}^{+}$ion beam. To determine the dopant concentration in the samples, two calibration standards have been used: a $\mathrm{Si}$ sample and a NiSi sample with a known As concentration.

In order to determine the SBH Schottky diodes on n-type bulk (100) Si with a doping level of $1 \times 10^{15} \mathrm{~cm}^{-2}$ have been fabricated. Arsenic was implanted at $40 \mathrm{keV}$ to a dose of 3 $\times 10^{15} \mathrm{~cm}^{-2}$ into the back side of the Si wafer. Then, $300 \mathrm{~nm}$ thermal oxide was grown by wet oxidation at $1050{ }^{\circ} \mathrm{C}$ for $30 \mathrm{~min}$. Subsequently, optical lithography and reactive ion etching were used to open windows on the front side. Afterward, $2 \mathrm{keV}$ As at a dose of $1 \times 10^{15} \mathrm{~cm}^{-2}$ was implanted. After removal of the native oxide, $\mathrm{Ni}$ was deposited by e-beam evaporation. In the first annealing step at $280{ }^{\circ} \mathrm{C}$ for $90 \mathrm{~s}, \mathrm{Ni}_{2} \mathrm{Si}$ was formed and the unreacted $\mathrm{Ni}$ was removed. The second annealing step at $450{ }^{\circ} \mathrm{C}$ was then performed to form NiSi. Finally, aluminum was deposited on the heavily doped back side of the wafer.

In the following, we use the term "dopant slope" when referring to the slope of the As dopant profile at the $\mathrm{NiSi} / \mathrm{Si}$ interface into the $\mathrm{Si}$ substrate, given in $\mathrm{nm} / \mathrm{decade}$.

\section{ONE-STEP ANNEALING PROCESS FOR STEEP NISI/SI JUNCTIONS WITH DOPANT SEGREGATION}

In this section we discuss the dependence of the dopant profile at the $\mathrm{NiSi} / \mathrm{Si}$ interface on implantation energy, $\mathrm{NiSi}$ thickness, and implantation dose in the case of a one-step annealing process for NiSi formation.

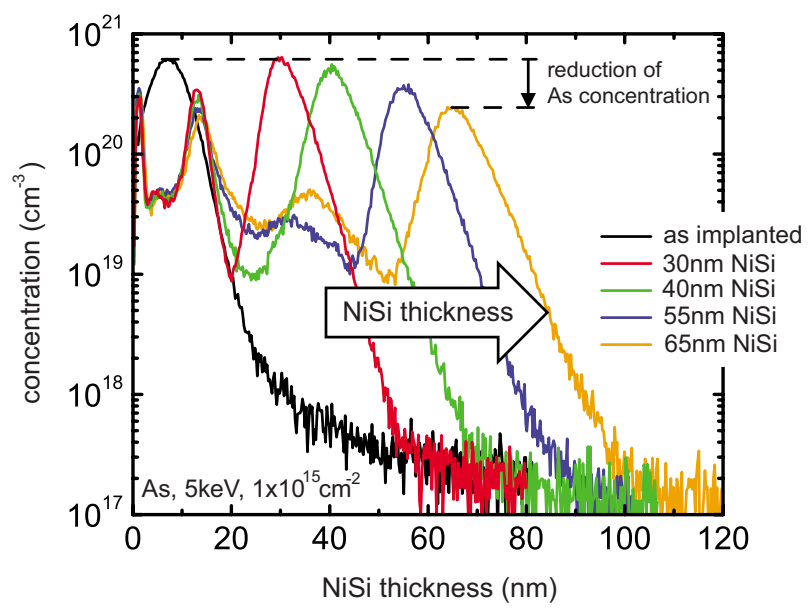

FIG. 2. (Color online) SIMS spectra of Si samples implanted with As, $5 \mathrm{keV}, 1 \times 10^{15} \mathrm{~cm}^{-2}$. The spectra of the as-implanted and silicided samples with different NiSi thicknesses are shown. The dopant peak shifts with the $\mathrm{NiSi} / \mathrm{Si}$ interface during silicidation. With the increase in the NiSi thickness, the peak concentration and the slope of the dopant distribution tail into $\mathrm{Si}$ decrease.

\section{A. Dependence of the dopant slope on implantation energy and NiSi thickness}

Figure 2 shows the SIMS spectra of a bulk Si sample implanted with As, $5 \mathrm{keV}, 1 \times 10^{15} \mathrm{~cm}^{-2}$ and of four identically implanted samples with different $\mathrm{NiSi}$ thicknesses ranging from 30 to $65 \mathrm{~nm}$. The peak shift of the dopant profile during silicidation with the movement of the $\mathrm{NiSi} / \mathrm{Si}$ interface confirms the dopant segregation process. ${ }^{17}$ The peak concentration decreases with increasing NiSi thickness from $6 \times 10^{20} \mathrm{~cm}^{-3}$ in the as-implanted case down to 2.3 $\times 10^{20} \mathrm{~cm}^{-3}$ for a NiSi thickness of $65 \mathrm{~nm}$. Simultaneously, the dopant slope increases from 6.8 to $10 \mathrm{~nm} /$ decade.

In Fig. 3(a) the slope of the dopant distribution is plotted versus the implantation energy for the as-implanted and silicided samples with NiSi thicknesses of 35 and $50 \mathrm{~nm}$. The graph shows that silicidation degrades the slope of the asimplanted dopant profile. For all implantation energies, a larger NiSi thickness results in a larger slope of the dopant distribution. Comparing the degradation of the slopes of the as-implanted dopant profiles through silicidation induced dopant segregation for 1 and $10 \mathrm{keV}$ implantation energies, we found that the decrease in slope for a steep initial profile is larger than for an initially broader dopant profile: 1.9 and 1.2 times, respectively.

The dopant slope degrades for each implantation energy in a similar way with increasing NiSi thickness, indicating the same mechanism for dopant redistribution for all implantation energies. This can be seen in Fig. 3(b) where the slope of the As profile is plotted as a function of the NiSi thickness. A large slope decrease is observed if either the implantation energy is increased or the thickness of the NiSi layer is increased. In the first case the degradation of the dopant slope is more severe compared to the one caused by dopant segregation because of different mechanisms: for the asimplanted case, the increased scattering with the host atoms with increasing energy causes a broadening of the dopant 

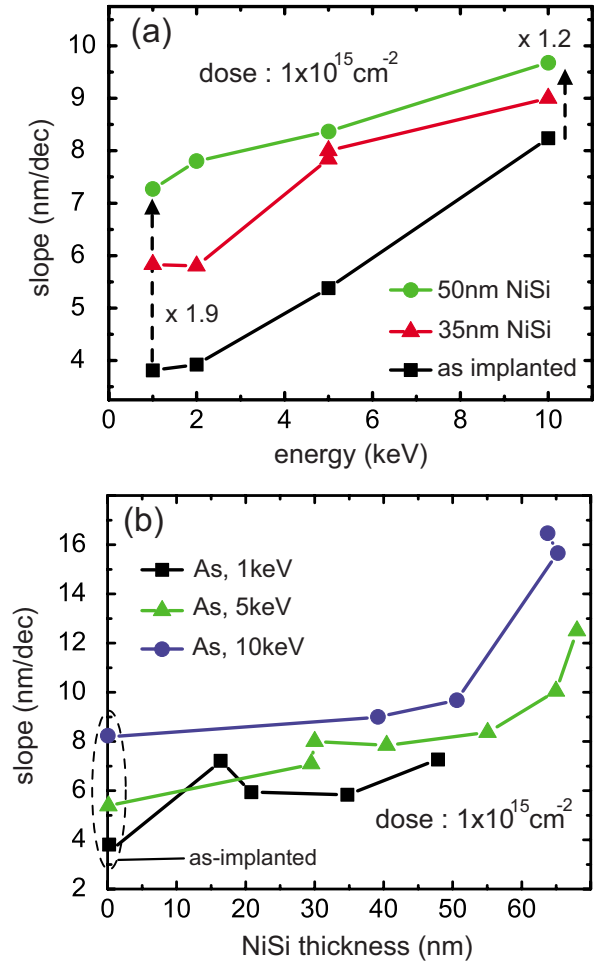

FIG. 3. (Color online) (a) Slope of the As dopant distribution tail into Si as a function of the implantation energy. The values of the as-implanted and of samples silicided after implantation are shown. (b) Slope of the As dopant distribution tail as a function of the NiSi thickness. With increasing NiSi thickness, the slope of the dopant distribution tail decreases. A steeper initial dopant profile for a lower implantation energy results in a steeper profile after silicidation. The dotted line connects the points corresponding to the as-implanted profiles.

profile, while for the silicided samples, redistribution of dopants between the forming $\mathrm{NiSi}$ and the $\mathrm{Si}$ bulk is responsible for the degraded slope.

As a result, since the degradation of the dopant slope due to segregation is significantly weaker compared to the effect of increased implantation energy, it is advantageous, for a fixed NiSi thickness, to choose a low implantation energy to optimize the steepness of the dopant profile after silicidation.

\section{B. Dependence of the dopant concentration at the $\mathrm{NiSi} / \mathrm{Si}$ interface on implantation energy and NiSi thickness}

Figure 4(a) displays the dopant concentration at the $\mathrm{NiSi} / \mathrm{Si}$ interface as a function of the NiSi thickness for implantation energies of $1,2,5$, and $10 \mathrm{keV}$. The dopant concentration in the as-implanted samples drops with increasing implantation energy from 1 to $10 \mathrm{keV}$ from the solid solubility limit of arsenic in silicon of $2 \times 10^{21}$ to $4 \times 10^{20} \mathrm{~cm}^{-3}$. ${ }^{18}$ The curves corresponding to implantation energies of 2,5 , and $10 \mathrm{keV}$ show a similar behavior with increasing NiSi thickness: the dopant concentration remains constant for a range of NiSi thicknesses before decreasing due to dopant diffusion into the silicon and the finite solubility of arsenic in $\mathrm{NiSi}$. Due to the low silicidation temperature of $500{ }^{\circ} \mathrm{C}$, outdiffusion of dopants can be ruled out. The concentration at the interface starts to decrease from the as-implanted value for a NiSi thickness of about three times the depth of the
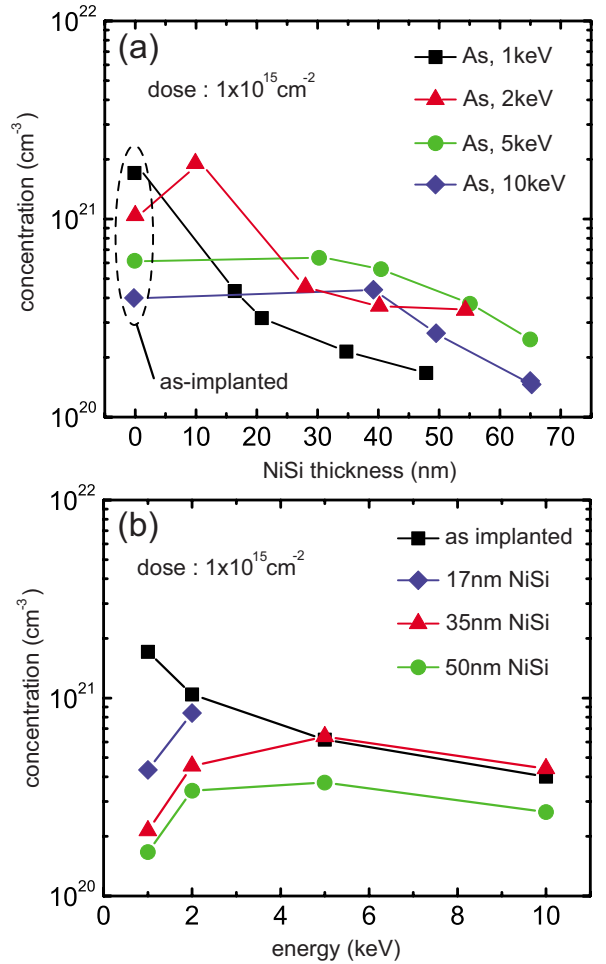

FIG. 4. (Color online) (a) As concentration at the $\mathrm{NiSi} / \mathrm{Si}$ interface as a function of the NiSi thickness for different implantation energies. The dotted line connects the points corresponding to the as-implanted samples. (b) As concentration at the $\mathrm{NiSi} / \mathrm{Si}$ interface as a function of the implantation energy for different NiSi thicknesses.

implantation peak. In the case of an implantation energy of $2 \mathrm{keV}$, the arsenic concentration decreases from 1 $\times 10^{21} \mathrm{~cm}^{-3}$ in the as-implanted sample to $8.5 \times 10^{20} \mathrm{~cm}^{-3}$ for a NiSi thickness of $17 \mathrm{~nm}$ and further to $4.5 \times 10^{20}$ and $3.5 \times 10^{20} \mathrm{~cm}^{-3}$ for NiSi thicknesses of 35 and $50 \mathrm{~nm}$, respectively. The apparently different behavior of the $1 \mathrm{keV}$ implanted sample compared to the other three energies arises from the fact that the thickness of the thinnest layer $(17 \mathrm{~nm})$ is a factor of 8 larger than the depth of the as-implanted concentration peak.

Following the same reasoning as in Sec. III A, we plot in Fig. 4(b) the dopant concentration at the $\mathrm{NiSi} / \mathrm{Si}$ interface as a function of the implantation energy. The as-implanted cases are plotted for comparison. For a fixed implantation energy, the As concentration decreases with increasing NiSi thickness, e.g., for $1 \mathrm{keV}$ with $\mathrm{NiSi}$ thicknesses from 17 to $50 \mathrm{~nm}$. The $17 \mathrm{~nm}$ NiSi layer is, as discussed above, too thick in order to maintain the as-implanted dopant concentration. However, at this NiSi thickness the interface dopant concentration is very close to the as-implanted value for the case of $2 \mathrm{keV}$ implantation energy.

Summarizing, for each implantation energy there is an optimum NiSi thickness (and vice versa for each NiSi thickness an optimum range of implantation energies) for which the dopant concentration at the $\mathrm{NiSi} / \mathrm{Si}$ interface reaches the as-implanted concentration peak value.

\section{Dependence of the dopant slope on implantation dose}

Figure 5(a) shows two SIMS profiles of samples implanted with arsenic at $5 \mathrm{keV}$ to doses of $5 \times 10^{13}$ and 1 


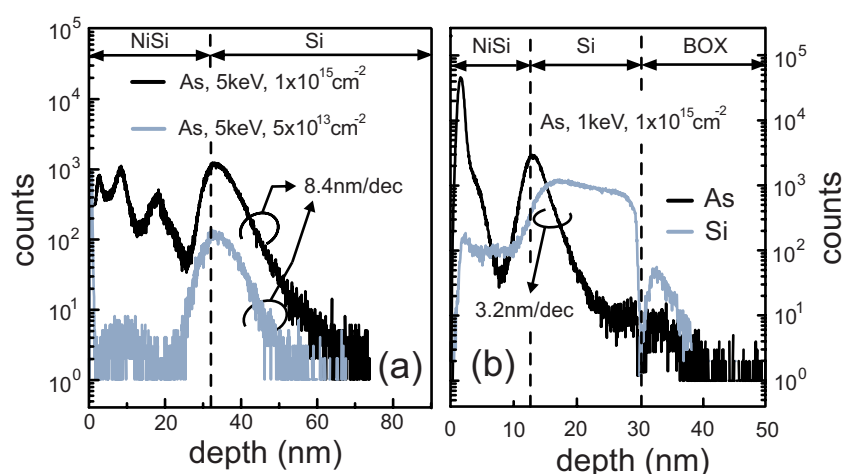

FIG. 5. (Color online) (a) SIMS profiles of bulk Si samples implanted with arsenic at $5 \mathrm{keV}$ to doses of $5 \times 10^{13}$ and $1 \times 10^{15} \mathrm{~cm}^{-2}$, respectively. After implantation, a $30 \mathrm{~nm} \mathrm{NiSi}$ layer was formed on both samples. (b) As and $\mathrm{Si}$ SIMS profiles of a $30 \mathrm{~nm}$ SOI sample implanted with As $1 \mathrm{keV}, 1$ $\times 10^{15} \mathrm{~cm}^{-3}$, and a $13 \mathrm{~nm}$ NiSi layer formed by a two-step annealing process. The slope of the dopant distribution at the $\mathrm{NiSi} / \mathrm{Si}$ interface is $3.2 \mathrm{~nm} /$ decade.

$\times 10^{15} \mathrm{~cm}^{-2}$, respectively. After implantation, both samples are silicided to a NiSi thickness of $30 \mathrm{~nm}$. The difference in the implantation dose translates into a different dopant concentration at the $\mathrm{NiSi} / \mathrm{Si}$ interface after segregation. Interestingly, the slope of the dopant profiles is $8.4 \mathrm{~nm} /$ decade for both concentrations. It has been shown previously by Zhang et al. that a high concentration of dopants at the $\mathrm{NiSi} / \mathrm{Si}$ interface is required for an efficient lowering of the $\mathrm{SBH}^{12}$ Therefore, it is advantageous from a device point of view to choose a high implantation dose.

\section{Summary and discussion}

The results on the formation of steep $\mathrm{NiSi} / \mathrm{Si}$ junctions with As dopant segregation discussed in the previous subsections can be summarized as follows: the smaller the initial implantation energy, the steeper will be the dopant profile at the NiSi/Si interface. At the same time, a small implantation energy also leads to a high peak concentration of dopants. For the generation of low SB NiSi contacts, it therefore seems beneficial to choose implantation energies as low as possible. However, in the limit of very low implantation energies dopants are pushed forward by the silicidation front during the whole silicidation process. This leads to a significant decrease in the dopant concentration at the $\mathrm{NiSi} / \mathrm{Si}$ interface once the silicide thickness becomes larger than roughly three times the depth of the initial implantation peak. As a result, for a certain targeted silicide thickness, the implantation energy has to be chosen in order to obtain the best trade-off between junction steepness and highest dopant concentration. For this the as-implanted peak has to be positioned close to the targeted NiSi thickness. The NiSi layer after silicidation should not be thicker than three times the as-implanted peak position.

\section{MODIFIED SILICIDATION PROCESS: DEPENDENCE OF JUNCTION STEEPNESS ON THE ANNEALING PROCESS AND THE ORDER OF IMPLANTATION AND NISI FORMATION}

In this section we present a modified annealing sequence that offers the possibility to obtain a steeper dopant profile at the $\mathrm{NiSi} / \mathrm{Si}$ interface than by employing the one-step process discussed in the previous section.

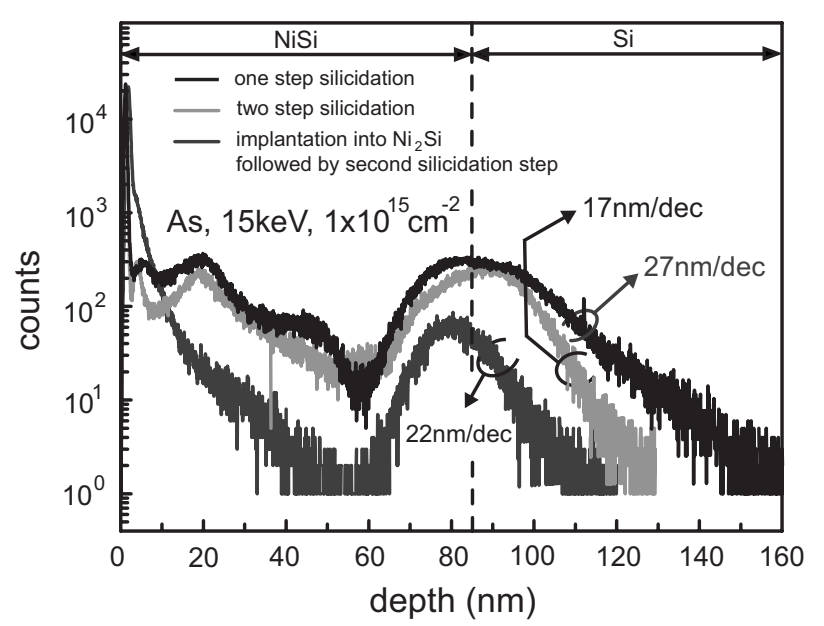

FIG. 6. Comparison of the SIMS profiles of As dopant distribution at the $\mathrm{NiSi} / \mathrm{Si}$ interface for three annealing processes. Implantation conditions are in all cases: As, $15 \mathrm{keV}, 1 \times 10^{15} \mathrm{~cm}^{-2}$. (a) One-step silicidation (black line), (b) two-step silicidation (light gray), and (c) implantation into $\mathrm{Ni}_{2} \mathrm{Si}$, followed by a second annealing to form NiSi.

Figure 5(b) shows the SIMS spectrum of a $13 \mathrm{~nm} \mathrm{NiSi}$ layer formed on a $30 \mathrm{~nm}$ SOI sample with a two-step silicidation process consisting of ion implantation (As, $1 \mathrm{keV}, 1$ $\times 10^{15} \mathrm{~cm}^{-2}$ ), annealing at $280{ }^{\circ} \mathrm{C}$ for $90 \mathrm{~s}$ to form $\mathrm{Ni}_{2} \mathrm{Si}^{19}$ removal of unreacted $\mathrm{Ni}$, and annealing at $500{ }^{\circ} \mathrm{C}$ to form $\mathrm{NiSi}$. In this case, the slope of the As dopant profile is $3.2 \mathrm{~nm} /$ decade in comparison to $5 \mathrm{~nm} /$ decade for the onestep annealing process. In Fig. 6, three differently processed samples implanted with As at an energy of $15 \mathrm{keV}$ to a dose of $1 \times 10^{15} \mathrm{~cm}^{-2}$ and $85 \mathrm{~nm} \mathrm{NiSi}$ are compared. On the first sample NiSi was formed after ion implantation with a onestep silicidation process. On the second sample NiSi was formed after implantation with the two-step silicidation process described above. As in the case of low implantation energy and small NiSi thickness, the slope of the dopant profile improves from $27 \mathrm{~nm} /$ decade for the one-step to $17 \mathrm{~nm} /$ decade for the two-step process. On the third sample $\mathrm{Ni}_{2} \mathrm{Si}$ was first formed by annealing at $280^{\circ} \mathrm{C}$ and subsequently, As implantation into the $\mathrm{Ni}_{2} \mathrm{Si}$ layer was performed. ${ }^{20}$ Finally, $\mathrm{Ni}_{2} \mathrm{Si}$ was transformed to $\mathrm{NiSi}$ through a second annealing step at $500{ }^{\circ} \mathrm{C}$. The slope of the dopant profile for this process is $22 \mathrm{~nm} /$ decade. While there is an improvement in dopant slope compared to one-step annealing, the dopant concentration at the $\mathrm{NiSi} / \mathrm{Si}$ interface is reduced by nearly an order of magnitude compared to the two other processes. This is disadvantageous since a high interfacial dopant concentration is imperative for an efficient lowering of the $\mathrm{SBH}^{7}$

We therefore conclude that the sharpest dopant slope with the largest dopant concentration is obtained by a twostep silicidation process for NiSi formation after ion implantation.

\section{SB LOWERING BY TWO-STEP SILICIDATION WITH DOPANT SEGREGATION AFTER IMPLANTATION}

In this section, we demonstrate that the two-step annealing process for the formation of $\mathrm{NiSi} / \mathrm{Si}$ junctions with $\mathrm{As}$ dopant segregation is effective in lowering the SBH. 

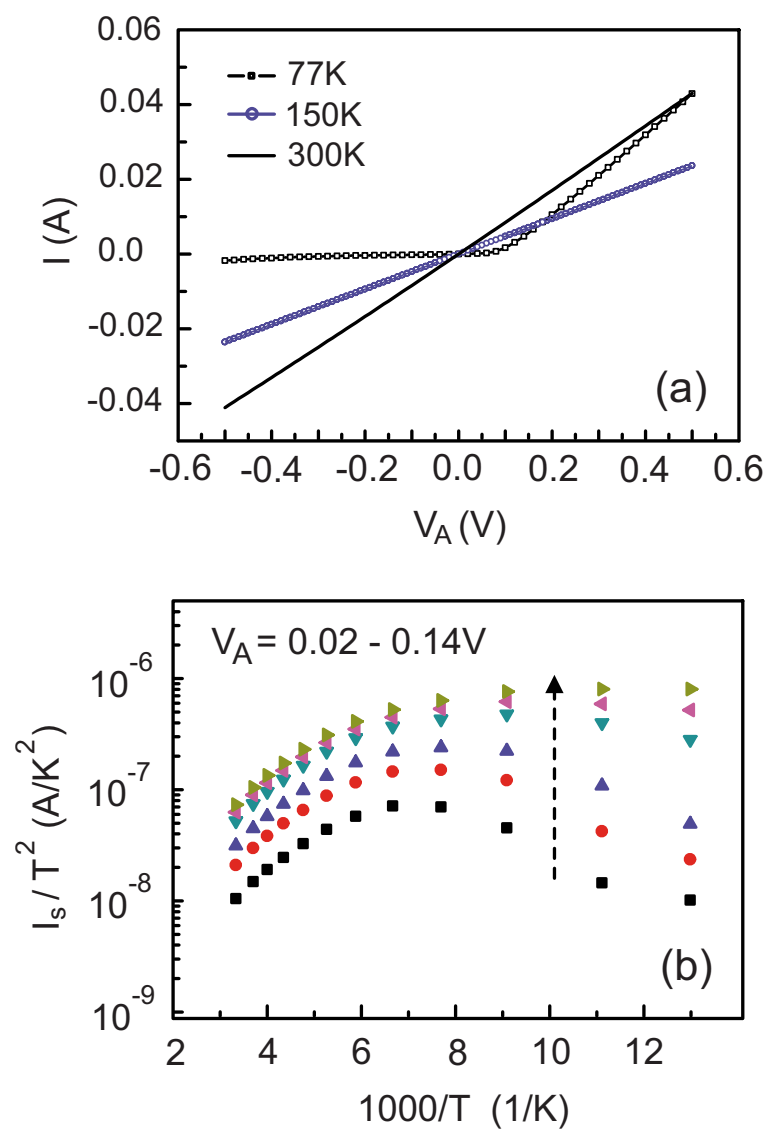

FIG. 7. (Color online) (a) $I-V$ characteristics of a NiSi Schottky diode with dopant segregation for different temperatures. Before two-step silicidation the silicon was implanted with As, $2 \mathrm{keV}, 1 \times 10^{15} \mathrm{~cm}^{-2}$. (b) $I_{\mathrm{S}} / T^{2}$-Arrhenius plot for the extraction of the effective SBH under different applied biases $\left(V_{\mathrm{A}}=0.02-0.14 \mathrm{~V}\right)$.

In order to determine the $\mathrm{SBH}$ at the $\mathrm{NiSi} / \mathrm{Si}$ interfaces, temperature dependent $I-V$ measurements of Schottky diodes have been performed. Before silicidation, the diodes were implanted with As, $2 \mathrm{keV}, 1 \times 10^{15} \mathrm{~cm}^{-2}$. A $20 \mathrm{~nm} \mathrm{NiSi}$ layer was formed afterward by the two-step annealing process described above. Figure 7(a) shows the current-voltage $(I-V)$ characteristics at temperatures of $T=77,150$, and $300 \mathrm{~K}$ of a NiSi Schottky diode with dopant segregation formed by two-step silicidation. Down to a temperature of $150 \mathrm{~K}$, an ohmic $I-V$ curve is measured. At a temperature of $77 \mathrm{~K}$, the diode shows rectifying behavior, an indication that a fairly large SB still exists. The change in slope under forward bias with temperature is due to the temperature dependence of the series resistance. According to thermal emission theory, the reverse current of a SB diode should saturate at a constant value but the experimental curve shows a slight increase in the reverse current with reverse bias. This could be caused by the Schottky effect or electrons tunneling through the SB from the metal into the semiconductor.

Figure 7(b) shows Arrhenius plots of the diode for the applied biases of $V_{\mathrm{A}}=0.02-0.14 \mathrm{~V}$. The extracted SBH varies between $\Phi_{\mathrm{SB}}=0.10-0.14 \mathrm{eV}$, depending on the forward bias and temperature range used for the extraction. This SBH extraction method does not take into account the effect of series resistance and may lead to errors if the series resistance is larger than the resistance of the SB. ${ }^{21}$ Therefore, we

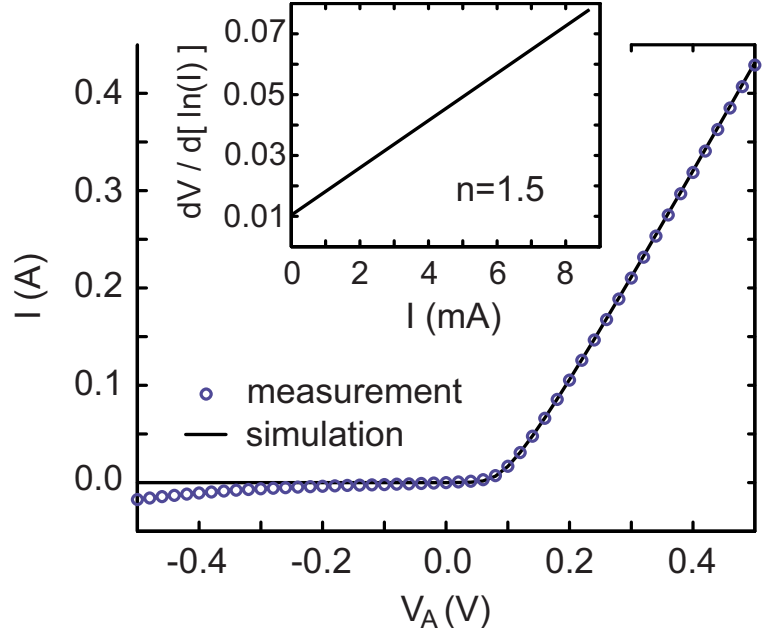

FIG. 8. (Color online) Experimental (dotted) and simulated $I-V$ curve at a temperature of $T=77 \mathrm{~K}$ of the NiSi Schottky diode with As dopant segregation formed by two-step annealing. From the comparison of simulation and experiment in the forward bias region, the series resistance was determined to $R_{\mathrm{s}}=8.7 \Omega$. The inset shows the extraction of the ideality factor to $n=1.5$.

also determined the SBH from a simulation of the $I-V$ characteristic of the SB diode taking a series resistance of $R_{\mathrm{S}}$ $=8.7 \Omega$ into account. The series resistance has been determined from the forward bias region of the measured $I-V$ characteristic. The $I-V$ curve has been calculated according to the formula ${ }^{22}$

$$
I=A^{*} A T^{2} \exp \left(\frac{q \Phi_{S B}}{k_{\mathrm{B}} T}\right)\left\{\exp \left[\frac{q\left(V-I R_{\mathrm{S}}\right)}{n k_{\mathrm{B}} T}\right]-1\right\},
$$

where $A^{*}$ is the Richardson constant, $A$ is the diode area, $T$ is the temperature, $q$ is the electric charge of the electron, $k_{\mathrm{B}}$ is the Boltzmann constant, $n$ is the ideality factor, and $V$ is the applied bias. Figure 8 shows the experimental $I-V$ curve measured at a temperature of $T=77 \mathrm{~K}$ and the simulated $I-V$ curve. The ideality factor $n$ was determined from the y-axis intercept using ${ }^{23}$

$$
\frac{d V}{d(\ln I)}=n \frac{k_{\mathrm{B}} T}{q}+I R_{\mathrm{S}}
$$

to be $n=1.5$. The $\mathrm{SBH}$ was determined from the simulations to $\Phi_{\mathrm{SB}}=0.12 \mathrm{eV}$ taking the series resistance and the nonideality of the diode into account. This demonstrates efficient SBH lowering by two-step silicidation.

More information on the measurement of SBH especially with back-to-back diodes can be found in Refs. 23 and 24.

\section{CONCLUSION}

We experimentally studied arsenic dopant profiles at the $\mathrm{NiSi} / \mathrm{Si}$ interface formed by silicidation induced dopant segregation. It was found that segregation causes a reduction in the dopant concentration at the $\mathrm{NiSi} / \mathrm{Si}$ interface from the as-implanted value for $\mathrm{NiSi}$ thicknesses larger than three times the as-implanted dopant distribution peak position. The slope of the dopant profile into $\mathrm{Si}$ at the $\mathrm{NiSi} / \mathrm{Si}$ interface degrades due to segregation for all NiSi thicknesses. This 
degradation is small compared to the decrease in slope caused by an increase in implantation energy. The largest slope and highest dopant concentration at the NiSi/Si interface are therefore obtained for low implantation energies and thin NiSi layers. We have demonstrated that improvements of the dopant slope can be obtained if the NiSi layer is formed with a two-step annealing sequence after ion implantation. For As, $1 \mathrm{keV}, 1 \times 10^{15} \mathrm{~cm}^{-2}$, and a $17 \mathrm{~nm} \mathrm{NiSi}$ layer, a NiSi/Si junction with a dopant slope of $3.2 \mathrm{~nm} /$ decade was obtained. Finally, the SBH of a $20 \mathrm{~nm}$ NiSi Schottky diode with arsenic dopant segregation formed with a two-step annealing process was determined to $\Phi_{\mathrm{SB}}=0.12 \mathrm{eV}$. These results show that with the technique of silicidation induced dopant segregation steep and shallow junctions with low $\mathrm{SBH}$ can be formed as required in nanoscale SOI SB-FETs.

\section{ACKNOWLEDGMENTS}

The authors thank their former colleague Dr. M. Zhang for discussions and his valuable comments.

This research has received Nanosil funding from the European Community (FP7, Grant No. 216171) and from the German Federal Ministry of Education via the MEDEA+ project DECISIF (Grant No. 2T104).

${ }^{1}$ ITRS International Technology Roadmap for Semiconductors, http:// public.itrs.net/.

${ }^{2}$ J. Kedzierski, P. Xuan, V. Subramanian, J. Bokor, T. J. King, and C. Hu, Tech. Dig. - Int. Electron Devices Meet. 2000, 57.

${ }^{3}$ Y. Wu, J. Xiang, C. Yang, W. Lu, and C. Lieber, Nature (London) 430, 61 (2004).

${ }^{4}$ J. Guo and M. S. Lundstrom, IEEE Electron Device Lett. 49, 1897 (2002).

${ }^{5}$ M. Jang, J. Oh, S. Maeng, W. Cho, S. Lee, K. Kang, and K. Park, Appl.
Phys. Lett. 83, 2611 (2003).

${ }^{6}$ G. Larrieu and E. Dubois, IEEE Trans. Electron Devices 52, 2720 (2005).

${ }^{7}$ M. Zhang, J. Knoch, Q. T. Zhao, St. Lenk, U. Breuer, and S. Mantl, European Solid-State Device Research Conference, Conference Digest, 2005 (unpublished), p. 457.

${ }^{8}$ A. Kinoshita, Y. Tsuchiya, A. Yagishita, K. Uchida, and J. Koga, 2004 Symposium on VLSI Technology, 2004 (unpublished), p. 168.

${ }^{9}$ Z. Zhang, Z. J. Qiu, P.-E. Hellström, G. Malm, J. Olsson, J. Lu, M. Östling, and S.-L. Zhang, IEEE Electron Device Lett. 29, 125 (2008).

${ }^{10}$ Q. T. Zhao, U. Breuer, E. Rije, St. Lenk, and S. Mantl, Appl. Phys. Lett. 86, 062108 (2005)

${ }^{11}$ J. Knoch, M. Zhang, S. Mantl, and J. Appenzeller, IEEE Trans. Electron Devices 53, 1669 (2006).

${ }^{12}$ M. Zhang, J. Knoch, J. Apenzeller, and S. Mantl, IEEE Electron Device Lett. 28, 223 (2007).

${ }^{13}$ C. Urban, C. Sandow, Q.-T. Zhao, and S. Mantl, Proceedings of the Tenth International Conference on Ultimate Integration of Silicon, Aachen, 2009 (unpublished), p. 65.

${ }^{14}$ G. Larrieu, E. Dubois, R. Valentin, N. Breil, F. Danneville, G. Dambrine, J. P. Raskin, and J. C. Pesant, Tech. Dig. - Int. Electron Devices Meet. 2007, 147.

${ }^{15}$ S. M. Sze and K. K. Ng Physics of Semiconductor Devices, 3rd ed. (Wiley-Interscience, New York, 2007).

${ }^{16}$ J. Knoch, M. Zhang, J. Apenzeller, and S. Mantl, Appl. Phys. A: Mater. Sci. Process. 87, 351 (2007)

${ }^{17}$ M. Wittmer and K. N. Tu, Phys. Rev. B 29, 158 (1984).

${ }^{18} \mathrm{R}$. Hull, Properties of Crystalline Silicon (Institution of Electrical Engineers, London, UK 1999).

${ }^{19}$ Properties of Metal Silicides, edited by K. Maex and M. van Rossum (Institution of Electrical Engineers, London, UK 1995).

${ }^{20}$ Z. Zhang, Z. Qiu, R. Liu, M. Östling, and S.-L. Zhang, IEEE Electron Device Lett. 28, 565 (2007)

${ }^{21}$ K. Sato and Y. Yasumura, J. Appl. Phys. 58, 3655 (1985).

${ }^{22} \mathrm{H}$. Lüth, Surfaces and Interfaces of Solid Materials (Springer, Berlin, 1995).

${ }^{23}$ E. H. Rhoderick and R. H. Williams, Metal-Semiconductor Contacts (Oxford Science, Oxford, UK 1988).

${ }^{24}$ E. Dubois and G. Larrieu, J. Appl. Phys. 96, 729 (2004). 